\title{
Endoscopic mucosal resection with an additional working channel $(E M R+)$ in a porcine ex vivo model: a novel technique to improve en bloc resection rate of snare polypectomy
}

(9) $\odot \ominus$

\author{
Authors \\ Richard F. Knoop*, Edris Wedi", Golo Petzold, Sebastian C.B. Bremer, Ahmad Amanzada, Volker Ellenrieder, Albrecht \\ Neesse, Steffen Kunsch
}

Institution

Department of Gastroenterology and Gastrointestinal Oncology, University Medical Center, Goettingen, Germany

submitted 13.2.2019

accepted after revision 14.6.2019

Bibliography

DOI https://doi.org/10.1055/a-0996-8050 |

Endoscopy International Open 2020; 08: E99-E104

(c) Georg Thieme Verlag KG Stuttgart · New York elSSN 2196-9736

Corresponding author

Steffen Kunsch, Department of Gastroenterology and GI Oncology, University Medical Center Goettingen, Georg-

August-University, D-37075 Goettingen, Germany

Fax: +0495513966327

steffen.kunsch@med.uni-goettingen.de

\section{ABSTRACT}

Background and study aims Recently, a new external additional working channel (AWC) was introduced by which conventional endoscopic mucosal resection (EMR) can be improved to a technique termed "EMR+". We first evaluated this novel technique in comparison to classical EMR in flat lesions.

Methods The trial was prospectively conducted in an ex vivo animal model with porcine stomachs placed into the EASIE-R simulator. Prior to intervention, standardized lesions were set by coagulation dots, measuring 1, 2, 3 or $4 \mathrm{~cm}$.

Results Overall, 152 procedures were performed. EMR and $\mathrm{EMR}+$ were both very reliable in 1-cm lesions, each showing en bloc resection rates of $100 \%$. EMR+ en bloc resection rate was significantly higher in $2-\mathrm{cm}$ lesions $(95.44 \%$ vs. $54.55 \%, P=0.02)$, in $3-\mathrm{cm}$ lesions $(86.36 \%$ vs. $18.18 \%, P<$ $0.01)$ and also in $4-\mathrm{cm}$ lesions $(60.00 \%$ vs. $0 \%, P<0.01)$. Perforations occurred only in EMR+ procedures in 4-cm lesions ( 3 of $20 ; 15 \%$ ).

Conclusions With its grasp-and-snare technique, EMR+ facilitates en bloc resection of larger lesions compared to conventional EMR. In lesions $2 \mathrm{~cm}$ and larger, EMR+ has demonstrated advantages, especially concerning en bloc resection rate. At $3 \mathrm{~cm}$, EMR+ reaches its best discriminatory power whereas EMR+ has inherent limits at $4 \mathrm{~cm}$ and in lesions of that size, other techniques such as ESD or surgery should be considered.

\section{Introduction}

Endoscopic mucosal resection (EMR) is a well-established, safe and cost-effective technique for endoscopic treatment of precancerous gastrointestinal lesions and early-stage cancer [1, 2]. It features a rapid procedure time, a low risk of adverse events (AEs) and a relatively low technical complexity [3]. However, when it comes to larger lesions, EMR is associated with a decreasing rate of en bloc resections, resulting in increasing rates of recurrence. Particularly for large sessile or laterally spreading polyps $\geq 2 \mathrm{~cm}$, en bloc EMR is technically almost impossible [4]. At that stage, en bloc resection of colorectal lesions can only be achieved in about $30 \%$ of cases [3,5]. Alterna-

* These authors contributed equally. tively, EMR with a piecemeal technique can be performed, but it is associated with higher recurrences rates compared to successful en bloc resection [4].

Endoscopic submucosal dissection (ESD) is a sophisticated endoscopic technique by which complex and larger lesions can be successfully addressed [6]. ESD was initially developed in Japan for en bloc resection of flat gastric neoplasias [7, 8]. Today, ESD is also increasingly employed in the esophagus and for colorectal lesions [2,9]. Especially for resection of laterally spreading polyps and flat lesions $\geq 2 \mathrm{~cm}$, ESD offers a reliable and minimally invasive method.

ESD has several disadvantages, however, as it is associated with a not insignificant rate of AEs, particularly a higher rate of perforations, although most of them are microperforations [3]. 
Furthermore, it demands more time, costs and resources, and is technically complex even for experienced endoscopists. To date, there has been no broad application in the Western world of ESD and its use is mainly limited to expert centers.

Therefore, novel endoscopic techniques are needed which can be clinically implemented for en bloc resections of lesions $\geq 2 \mathrm{~cm}$ that are feasible and safe to use without extensive training.

Meeting the mentioned requirements, classical EMR technique can be improved with a new external additional working channel (AWC, Ovesco Endoscopy, Tuebingen, Germany) that was recently introduced and termed "EMR+ technique" [10]. To this end, the AWC is mounted on a standard endoscope similar to the setup used with the full-thickness resection device (FTRD) [11].

So far, EMR+ has not been systematically evaluated. Therefore, we provide the first data in terms of usefulness and feasibility of this novel method. The aim of the current study was to prospectively compare the novel technique EMR+ to the gold standard of classical EMR using a preclinical ex vivo porcine animal model. We chose en bloc resection rate, procedure time, and resection area in consideration of the complication rate as clinical endpoints to assess for which lesions EMR+ would be particularly appropriate.

\section{Materials and methods}

The study was designed as a prospective ex vivo trial. It was exempt from IRB review because no humans or living animals were included. The experiments were conducted at the research unit of the Department of Gastroenterology and Gastrointestinal Oncology, University Medical Center Goettingen, Germany.

Pig stomachs were cleaned and frozen. Prior to the procedure, they were defrosted and placed into the EASIE-R simulator (Endosim, LLC, Hudson, Massachusetts, United States). The EASIE-R stimulator is an established model for research and interventional endoscopic training and has already been evaluated for several endoscopic procedures $[12,13]$.
All interventions (EMR and EMR+) were performed by two well-trained endoscopists with previous experience in EMR and $\mathrm{EMR}+$ technique in humans and animal models.

\section{Additional working channel}

The AWC ( $\triangleright$ Fig. 1) has a flexible attachment, a shaft with a length of $122 \mathrm{~cm}$ (endoscope insertion length: $103-110 \mathrm{~cm}$ ), an adaptor for fixation at the endoscope handle with Luerlock, a valve and a sleeve with adhesive tape. The AWC can be mounted on endoscopes with a diameter from 8.5 to 13.5 $\mathrm{mm}$. Instruments with an outer diameter of up to $2.8 \mathrm{~mm}$ can be introduced. All AWC procedures were performed with the AWC in the counterpart position to the working channel.

\section{EMR and EMR+ procedure}

We selected standardized lesions, measuring $1 \mathrm{~cm}, 2 \mathrm{~cm}, 3 \mathrm{~cm}$ or $4 \mathrm{~cm}$. Prior to intervention, lesions in the corpus of the porcine stomach were manually marked with coagulation dots inside a template. Then, the stomach was transferred into the EASIE-R model. The porcine esophagus and stomach were fixed to the model's plastic shell [13].

After submucosal injection (hydroxyethyl starch with methylene blue), in all resections, a 33-mm snare (Boston Scientific, Malborough, Massachusetts, United States) was positioned around the lesion. A grasper (Ovesco Endoscopy, Tuebingen, Germany) was used for EMR+, which was introduced via the AWC. This can also be set the other way with the snare introduced via the original working channel and the grasper in the AWC as demonstrated in > Fig. $\mathbf{1}$.

If adequate positioning of a lesion required more than 15 minutes, the procedure was stopped and results were classified as failed en bloc resection. Fig. 2 illustrates the use of the $E M R+$ technique and a resected specimen in the ex-vivo model.

\section{Data collection}

The following parameters were recorded by an independent observer: Prepared lesion size $(1 \mathrm{~cm}, 2 \mathrm{~cm}, 3 \mathrm{~cm}$ or $4 \mathrm{~cm})$, rate of en bloc resection ( $R 0$, defined by all marking dots retrieved

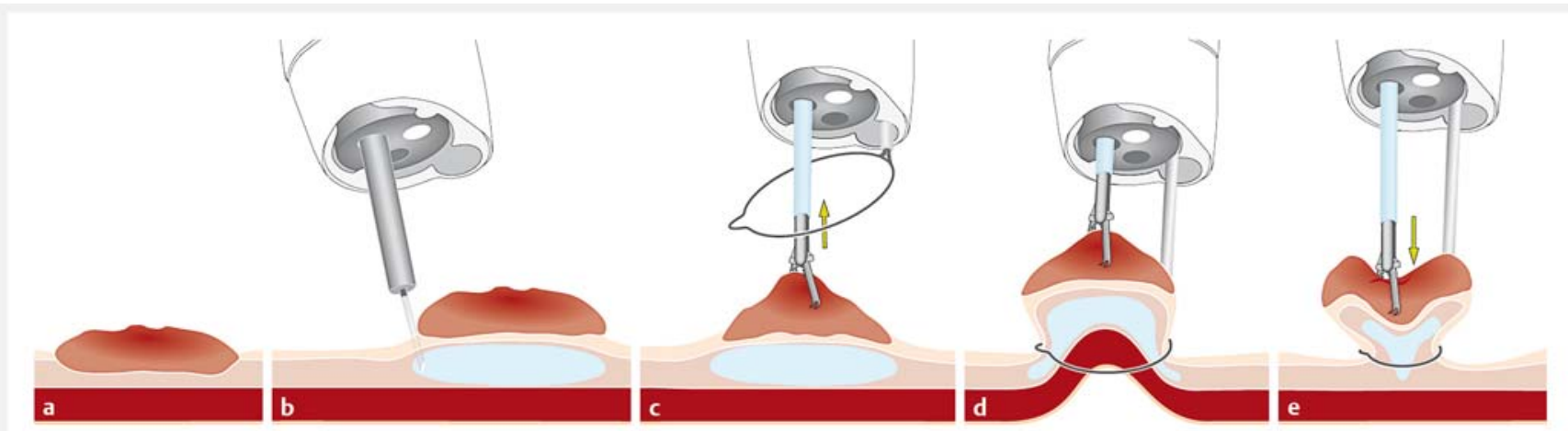

- Fig. 1 Principles of the EMR+ procedure. a Target lesion. b Submucosal injection. c Positioning of snare and grasper. $\mathbf{d}$ Elevation of the lesion and snare closure. e Pushback of the grasper while snare stays closed followed by resection. (Source: with permission from Ovesco Endoscopy AG, Tuebingen, Germany) 

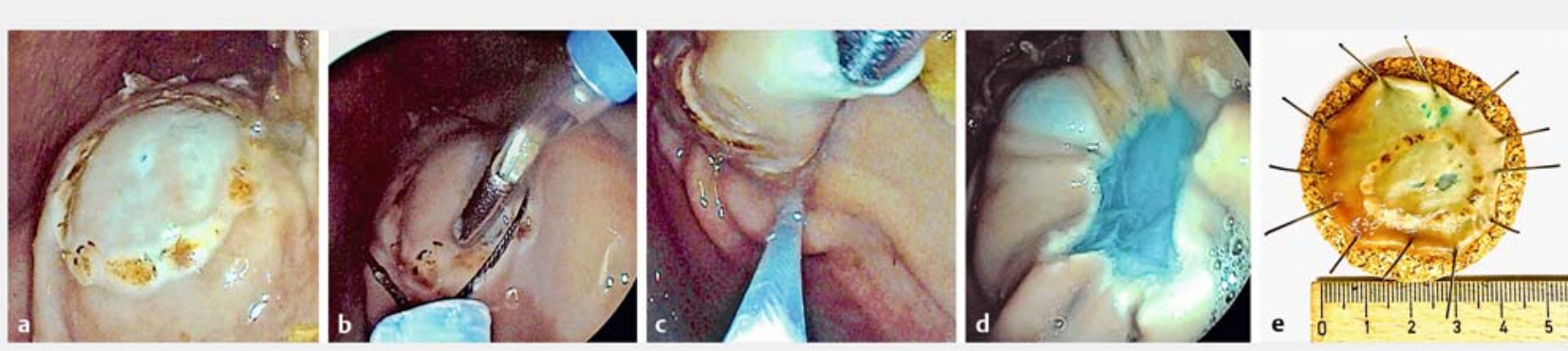

- Fig. 2 Application of EMR+ in the ex-vivo model. a Lesion to be targeted after submucosal injection. b Positioning of snare and grasper. c Elevation of the lesion and snare closure. $\mathbf{d}$ Post-interventional site of resection. $\mathbf{d}$ Resected specimen after EMR+.

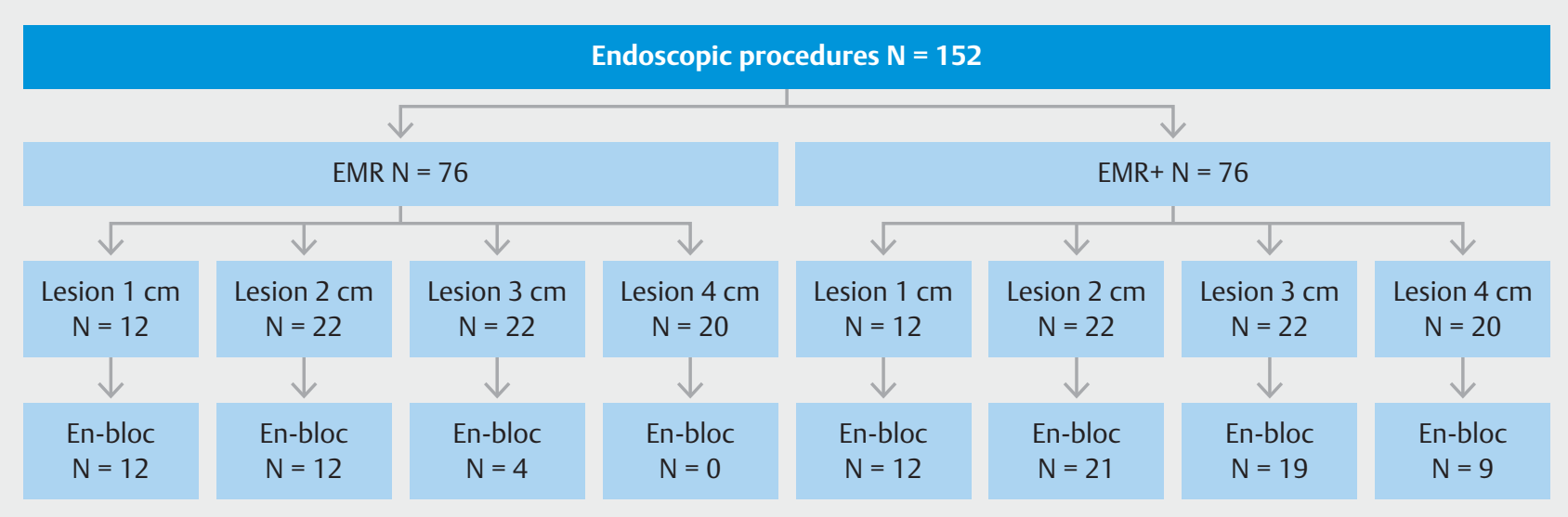

Fig. 3 Study design.

on the resected specimen), time of EMR and EMR+ procedure, resection area (in $\mathrm{cm}^{2}$ ), AEs (perforations).

After each EMR and EMR+, the specimens were spread out, pinned on cork plates and photographed. EMR and EMR+ procedure time was defined from submucosal injection to complete resection of the lesion. Following visual evaluation of each resection site, an insufflation test was performed to determine the presence of a perforation.

\section{Statistical analysis}

Data analysis was performed using SPSS (IBM). Chi-square-test was used for statistical analysis of en bloc resection rates. Mann-Whitney-U-Test was used for the analysis of time of procedure and resection area. $P<0.05$ was considered statistically significant and is marked by *.

\section{Results}

In both the EMR and EMR+ groups, lesions with four different sizes were set with a diameter of $1 \mathrm{~cm}$ ( $n=12$ per group), $2 \mathrm{~cm}$ ( $n=22$ per group), $3 \mathrm{~cm}(n=22$ per group) and $4 \mathrm{~cm}(n=20$ per group). In total, 152 endoscopic procedures (76 EMR, 76 EMR+) were performed in the ex vivo porcine model by two experienced endoscopists. Overall, we used 15 stomachs, each with eight to 12 lesions, dependent on stomach and lesion sizes. An overview of the study design is presented in - Fig. $\mathbf{3}$.

\section{Rate of en bloc resection}

In 1-cm lesions, EMR achieved an en bloc resection rate (R0) of $100 \%$ (12 of 12). In 2-cm lesions, it was $54.55 \%$ (12 of 22 ), decreasing to $18.18 \%$ (4 of 22 ) in $3-\mathrm{cm}$ lesions and ending with $0 \%$ ( 0 of 20 ) in $4-\mathrm{cm}$ lesions. In $1-\mathrm{cm}$ lesions, EMR+ resulted in an en bloc resection rate of $100 \%$ (12 of 12). Compared to EMR, $\mathrm{EMR}+$ en bloc resection rate was significantly higher in $2-\mathrm{cm}$ lesions ( $95.44 \%$ (21 of 22 ) vs. $54.55 \%, P=0.02 *)$, in $3-\mathrm{cm}$ lesions (86.36\% (19 of 22$)$ vs. $\left.18.18 \%, P<0.01^{*}\right)$ and also in $4-\mathrm{cm}$ lesions $\left(60.00 \%(12\right.$ of 20$)$ vs. $\left.0 \%, P<0.01^{*}\right)$. These data are presented in > Fig. 3 and $>$ Fig. 4.

\section{Procedure time}

In 1-cm lesions, median procedure time in the EMR-group was 2.50 minutes (SD 2.45). In 2-cm lesions, it was 6.00 minutes (SD 2.62), in 3-cm lesions, it was 12.50 minutes (SD 3.89) and in 4-cm lesions, median procedure time was 15.00 minutes (SD $0.58)$.

The difference in procedure times with EMR+ in 1-cm and 2$\mathrm{m}$ lesions did not reach significance in the statistical analysis (median 2.50 minutes (SD 0.90) vs. 2.50 minutes (SD 2.45, $P=$ 0.74 ; median 4.00 minutes (SD 2.34) vs. 6.00 minutes (SD 


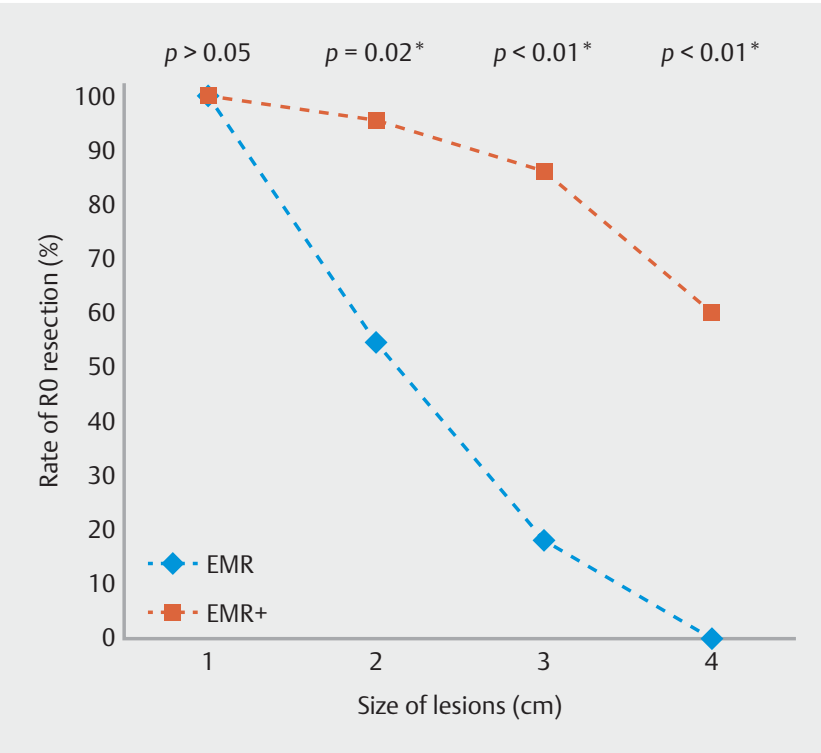

> Fig. 4 Rate of en bloc resection (R0).

2.62), $P=0.16)$. In contrast, procedure time in $3-\mathrm{cm}$ and $4-\mathrm{cm}$ lesions was significantly lower in the EMR+ group compared to EMR (5.00 minutes (SD 3.38) vs. 12.50 minutes, $P<0.01^{*} ; 5.50$ minutes (SD 2.69) vs. 15.00 minutes, $P<0.01^{*}$ ). These findings are presented in $\mathbf{~ F i g . ~} \mathbf{5}$.

\section{Resection area}

Median EMR resection area for $1-\mathrm{cm}$ lesions was $3.14 \mathrm{~cm}^{2}$ (SD 1.19). In $2-\mathrm{cm}$ lesions, it was $3.30 \mathrm{~cm}^{2}$ (SD 1.55). At $3 \mathrm{~cm}$ it was $1.50 \mathrm{~cm}^{2}$ (SD 2.81) and in 4-cm lesions, median resection area was $4.02 \mathrm{~cm}^{2}$ (SD 1.61) for EMR. In lesions of all sizes, the resection area was significantly larger in the EMR+ groups. In 1$\mathrm{cm}$ lesions, the median resection area was $4.44 \mathrm{~cm}^{2}$ for EMR+ (SD 1.56, $\left.P=0.012^{*}\right)$. It was $5.94 \mathrm{~cm}^{2}\left(\mathrm{SD} 3.91, P<0.01^{*}\right)$ at $2 \mathrm{~cm}$ and $9.62 \mathrm{~cm}^{2}$ (SD 3.98, $P<0.01^{*}$ ) at $3 \mathrm{~cm}$. In $4-\mathrm{cm}$ lesions, EMR+ median resection area was $13.37 \mathrm{~cm}^{2}\left(\mathrm{SD} 7.67, P=0.03^{*}\right)$ ( Fig.6).

\section{Perforations}

In all EMR groups, no perforations occurred, whereas in the $\mathrm{EMR}+$ group only in 4-cm lesions, three perforations were observed ( $15 \%, 3$ of 20$)$.

\section{Discussion}

With its broad availability, EMR is a well-established minimally invasive technique for treatment of dysplastic and early malignant gastrointestinal lesions. However, when it comes to treatment of large, particularly flat lesions, EMR has certain limitations.

For potentially challenging en bloc resection of flat lesions $\geq 2 \mathrm{~cm}$, ESD offers a reliable and oncologically convincing method [6]. But ESD comes with a not insignificant rate of AEs, especially perforations [3]. ESD is expensive, time-consuming and technically complex as it involves a long learning curve even

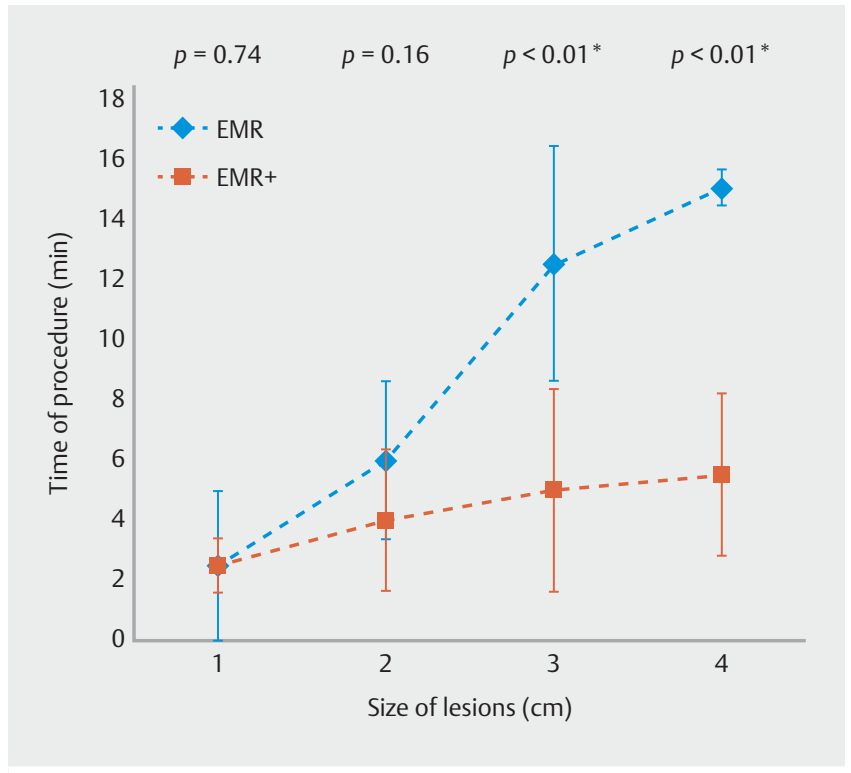

Fig. 5 Duration of EMR and EMR+ procedure.

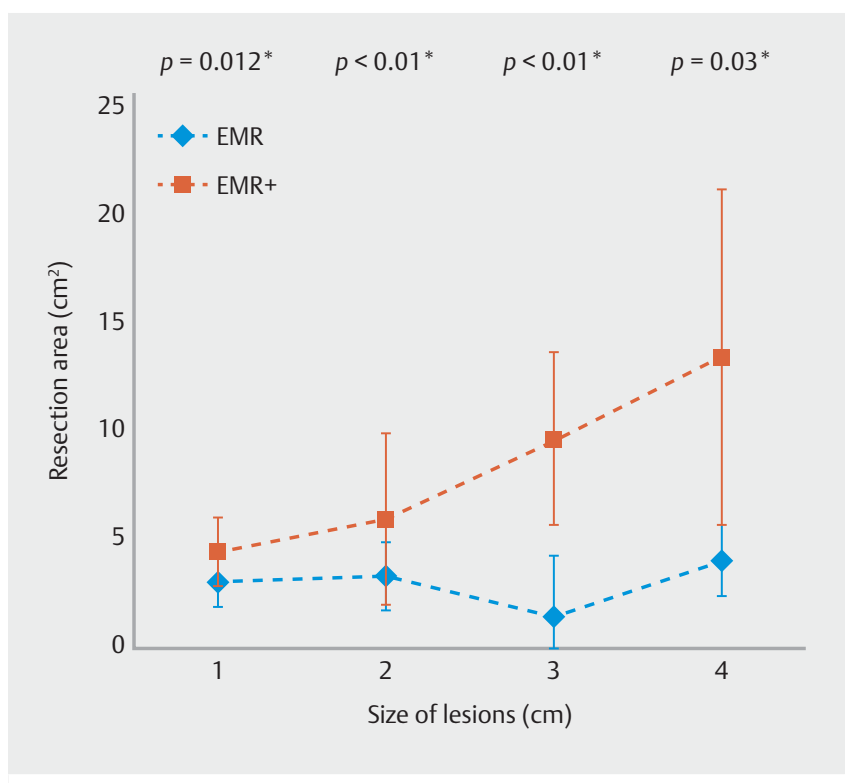

Fig. 6 Resection area (in $\mathrm{cm}^{2}$ ).

for experienced endoscopists [3]. For these reasons, in the Western world, ESD is mostly limited to expert centers [3]. EFTR has especially advanced management of complex colorectal precancerous and malignant lesions without lifting sign, e. g. due to scarring $[14,15]$. However, its applicability is practically limited to the lower gastrointestinal tract and its diameter is not appropriate for flat and spreading lesions, which are object of this study [16-18].

Bearing this in mind, EMR+ was recently developed and is based on an additional working channel. An effective grasp and snare technique using a dual-channel endoscope has already been described [19-21]. However, its practicality is limited due to the close and fixed distance between the two work- 
ing channels, which results in a lack of sufficient triangulation, flexibility, and overview. Furthermore, a dual-channel endoscope is an expensive investment for endoscopy units and is consequently not always available.

In analogy to the FTRD [11], the AWC is mounted at the tip of a standard gastroscope or pediatric colonoscope, thus making a dual-channel endoscope dispensable. By turning its cap, more variable and wider positions of both working channels (AWC plus standard channel) can be achieved in contrast to a dual-channel endoscope [10]. If required, this leads to better visibility and more flexible triangulation of the instruments.

First, the results of our prospective ex vivo study represent the frequently described advantages and limitations of classical EMR, e.g. implemented in the European guidelines [1]. In analogy to the clinical situation, also in our ex-vivo porcine animal model, EMR is very reliable for lesions of $1 \mathrm{~cm}$ with an en bloc resection rate of $100 \%$. For 2 -cm lesions, that drops to $55 \%$, whereas classical EMR does not provide satisfying resection rates for $3-\mathrm{cm}$ or $4-\mathrm{cm}$ lesions ( $18 \%$ and $0 \%$, respectively). Thus, our model seems to represent the clinical reality with a good discriminatory power in terms of the gold standard EMR, showing insufficient results in lesions $\geq 2$ to $4 \mathrm{~cm}$ [1].

In defined 1-cm lesions, there was no difference between $E M R$ and EMR+ in terms of en bloc resection rate. However, en bloc resection rates with conventional EMR decreased rapidly for defined 2-cm lesions. Lesions measuring $3 \mathrm{~cm}$ showed a poor en bloc resection rate for EMR compared to EMR+, the latter revealing satisfying results at $86 \%$. In $4-\mathrm{cm}$ lesions, the en bloc resection rate for EMR+ dropped to $60 \%$, whereas it was $0 \%$ in the EMR group. Obviously, 4-cm lesions basically cannot be addressed with conventional EMR. Hence in our animal model, the critical size of lesions to be successfully removed by conventional EMR corresponds to the data in humans [1, $3-$ 5].

Furthermore, procedure time was not longer if EMR+ was used. Conversely, starting at a size of $2 \mathrm{~cm}$, procedure time for lesions was significantly shorter in the EMR+ subgroups compared to conventional EMR. Based on the technical aspects of $\mathrm{EMR}+$, larger resection areas were achieved in all groups, starting with 1-cm lesions.

In 4-cm lesions, few perforations occurred in the EMR+ group. Because conventional EMR becomes completely insufficient in lesions of this size, they cannot be compared in terms of use of EMR versus EMR+. However, our data suggest that $4-\mathrm{cm}$ lesions also seem to be too big for EMR+.

Our prospective study was conducted in an established and well-evaluated ex vivo animal model. However, there are certain limitations that may limit the transferability from the porcine ex vivo model to humans. First, to ensure a standardized experimental setup, we consistently used a 33-mm snare for both EMR and EMR+ for all lesions. Snare size potentially affects en bloc resection rate. Second, the porcine stomach has higher mucosal rigidity than human gastric mucosa, which affects the technical opportunities for EMR and EMR+. Obviously, bleeding, tissue movement, histopathological evaluation and other physiological factors cannot be reproduced in our ex vivo mod- el. Principally, damage may occur to the specimen as a result of grabbing the lesion with the forceps.

Our data clearly demonstrate that EMR+ is a novel resection technique with potential to improve en bloc resection rates for snare-based polypectomy in flat lesions larger than $1 \mathrm{~cm}$. In terms of the high number of neoplastic flat lesions seen in the right colon, future reports on how AWC will work in difficult colonoscopy cases (tortuous sigmoid colon; severe diverticulosis; long, mobile, looping colons) are needed.

\section{Conclusion}

The newly developed EMR+ technique with AWC facilitates en bloc resection of larger lesions compared to conventional EMR, the well-described gold standard for lesions $<2 \mathrm{~cm}$. Consistently, we did not observe an additional benefit of EMR+ in these lesions whereas beginning at $2 \mathrm{~cm}$, EMR+ had advantages over conventional EMR, reaching its best discriminatory power over conventional EMR at $3 \mathrm{~cm}$. Because EMR+ reaches its inherent limits at $4 \mathrm{~cm}$, accompanied by a rising risk of perforations, ESD or surgery should be considered in lesions larger than that size.

In our ex-vivo porcine model, we could show that EMR+ works and can significantly and relevantly increase the size of lesions to be successfully resected en bloc. Consequently, using $\mathrm{EMR}+$, lesions can be resected en bloc that otherwise could only be addressed via ESD or surgery. Therefore, EMR+ could help to close a therapeutic gap with manageable technical complexity, time, and costs.

The AWC device allows easy transformation of a standard single-channel endoscope to double-channel functionality, leading to good opportunities for bimanual work by triangulation with better intraluminal resections accompanied by more efficient tissue traction. The AWC could also be used for other indications, e.g. for ESD procedures ("ESD+").

\section{Acknowledgements}

The authors thank Ovesco AG for providing figures, devices and equipment free of charge. They also would like to thank Boston Scientific for providing the used snares free of charge. They thank K. Salzmann and E. Herzog for assisting with the experiments.

\section{Competing interests}

Drs. Kunsch and Wedi conduct hands-on training for Ovesco AG.

\section{References}

[1] Ferlitsch M, Moss A, Hassan C et al. Colorectal polypectomy and endoscopic mucosal resection (EMR): European Society of Gastrointestinal Endoscopy (ESGE) Clinical Guideline. Endoscopy 2017; 49: 270-297 
[2] Wang J, Zhang XH, Ge J et al. Endoscopic submucosal dissection vs endoscopic mucosal resection for colorectal tumors: a meta-analysis. World J Gastroenterol 2014; 20: 8282 - 8287

[3] Holmes I, Friedland S. Endoscopic Mucosal resection versus endoscopic submucosal dissection for large polyps: a western colonoscopist's view. Clin Endosc 2016; 49: 454-456

[4] Meier B, Caca K, Fischer A et al. Endoscopic management of colorectal adenomas. Ann Gastroenterol 2017; 30: 592 - 597

[5] Zhan T, Hielscher T, Hahn F et al. Risk factors for local recurrence of large, flat colorectal polyps after endoscopic mucosal resection. Digestion 2016; 93: 311-317

[6] Pimentel-Nunes P, Dinis-Ribeiro M, Ponchon T et al. Endoscopic submucosal dissection: European Society of Gastrointestinal Endoscopy (ESGE) Guideline. Endoscopy 2015; 47: 829-854

[7] Ono S, Fujishiro M, Niimi K et al. Long-term outcomes of endoscopic submucosal dissection for superficial esophageal squamous cell neoplasms. Gastrointest Endosc 2009; 70: 860-866

[8] Nakamoto S, Sakai Y, Kasanuki J et al. Indications for the use of endoscopic mucosal resection for early gastric cancer in Japan: a comparative study with endoscopic submucosal dissection. Endoscopy 2009; 41: 746-750

[9] Tamegai $Y$, Saito $Y$, Masaki $N$ et al. Endoscopic submucosal dissection: a safe technique for colorectal tumors. Endoscopy 2007; 39: 418 422

[10] Wedi E, Knoop RF, Jung $C$ et al. Use of an additional working channel for endoscopic mucosal resection (EMR+) of a pedunculated sessile serrated adenoma in the sigmoid colon. Endoscopy 2019; 51: 279280

[11] Wedi E, Orlandini B, Gromski M et al. Full-thickness resection device for complex colorectal lesions in high-risk patients as a last-resort endoscopic treatment: initial clinical experience and review of the current literature. Clin Endosc 2018; 51: $103-108$
[12] Schöfl R, Buchmeier B, Hauder G. Adaptation of the Erlangen active simulator for interventional endoscopy (EASIE) model for transmural pancreatic pseudocyst drainage. Endoscopy 2006; 38: 100

[13] Hochberger J, Matthes K, Maiss J et al. Training with the compactEASIE biologic endoscopy simulator significantly improves hemostatic technical skill of gastroenterology fellows: a randomized controlled comparison with clinical endoscopy training alone. Gastrointest Endosc 2005; 61: 204-215

[14] Schmidt A, Bauerfeind P, Gubler $C$ et al. Endoscopic full-thickness resection in the colorectum with a novel over-the-scope device: first experience. Endoscopy 2015; 47: 719-725

[15] Knoop RF, Richer-Schrag HJ, Thimme R et al. Use of the full-thickness resection device (FTRD) to prevent bleeding after colonic polypectomy in a patient with a left ventricular assist device. Endosc Int Open 2017; 5: E749-E753

[16] Richter-Schrag H], Walker $C$, Thimme $R$ et al. [Full thickness resection device (FTRD) : Experience and outcome for benign neoplasms of the rectum and colon]. Chirurg 2016; 87: 316-325

[17] Schurr MO, Baur FE, Krautwald M et al. Endoscopic full-thickness resection and clip defect closure in the colon with the new FTRD system: experimental study. Surg Endosc 2015; 29: 2434-2441

[18] von Renteln D, Kratt T, Rosch T et al. Endoscopic full-thickness resection in the colon by using a clip-and-cut technique: an animal study. Gastrointest Endosc 2011; 74: 1108-1114

[19] von Renteln D, Schmidt A, Vassiliou MC et al. Endoscopic mucosal resection using a grasp-and-snare technique. Endoscopy 2010; 42: $475-480$

[20] Shetty A, Suarez AL, Dufault DL et al. Endoscopic mucosal resection with grasp-and-snare technique for challenging lesions. Gastrointest Endosc 2016; 84: 738 - 739

[21] de Melo SW Jr., Cleveland P, Raimondo M et al. Endoscopic mucosal resection with the grasp-and-snare technique through a doublechannel endoscope in humans. Gastrointest Endosc 2011; 73: $349-$ 352 